\title{
Psammophilic scorpions in deserts of Iran
}

Received: 23 December, 2020

Accepted: 18 May, 2021

Published: 19 May, 2021

*Corresponding author: Navidpour SH, Razi Reference Laboratory of Scorpion Research (RRLS), Razi Vaccine \& Serum Research Institute, Agricultural Research Education and Extension Organization (AREEO), Karaj, Iran, E-mail: navid1038@hotmail.com

Keywords: Faunestic; Psammopilus; Deserts scorpion; Iran

https://www. peertechz,com

\section{Check for updates}

\begin{abstract}
In this study totally 3453 scorpions belonged buthidae family collected from different localities in south and southwestern provinces of Iran. The six psammophilic scorpions were identified in this research: Kraepelinia palpator, Polisius persicus, Apistobuthus susanae , Buthacus macrocentrus, Vachoniolus iranus and Compsobuthus jakesi.
\end{abstract}

\section{Introduction}

The psammophilus scorpions are liked to life on desert area and this group of scorpions are stenotopes, maladapted to life outside of this environments, and are unviable to burrow soil [1-3].

Long tarsal claws and enlarged macrochaete setae arranged into "sand combs" increase the effective surface area in contact with the ground. This allows scorpions to walk on loose sand without sinking or loss of traction. Such leg morphology also allows efficient movement of sand during burrow construction $[2,3]$.

Psammophilic scorpions generally burrow to a depth of 03-1.om below the surface [3]. Some psammophiles (e.g., Vejovoidus longiunguis) have streamlined metasomas and telsons, which may aid in escape when animals become buried in the sand. Scorpions that encounter only sandy substates (ultrapsammophiles) may have greatly reduced pectines (Newlands 1972) [4]. Since pectines function in substrate discrimination daring spermatophore deposition, such hypotrophy may reflect their relative uselessness for scorpions that are in extremely homogeneous environments [4].

Farzanpay [5] and Fet, et al. [1,6] reported psammophilus genus Apisthobuthus and Buthacus from sandy regions of Khoozestan province and,Polisius persicus,from Sistan \& Bluchestan province (East of Iran).
The study was conducted for the first time in the Iranian desert scorpions and It shows the species diversity in the desert regions of Iran.

\section{Material and methods}

During this study from 2006 to 2012 specimens collected from different localities of six provinces of Iran: Khouzestan, Kerman, Fars, Ilam, Hormozgan and Boushehr. The specimens collected by night catch and UV light lamps.

\section{Results}

A total 3453 scorpions were collected during this study from different provinces of south and southwestern area in Iran which is from the highest to the minimum number of scorpions cached, as follows:

Apistobuthus susanae 1982 (57.40\%)

Buthacus macrocentrus 935 (27\%)

Compsobuthus jakesi 351 (10.16 \%)

Vachoniolus iranus 165 (4.8\%)

Polisius persicus $11(3.2 \%)$

Kraepelinia palpator $9(0.26 \%)$

\section{Kraepelinia palpator (Figures 1-4)}

Distribution: Iran, Kerman Province [7]; Turkmenistan. 

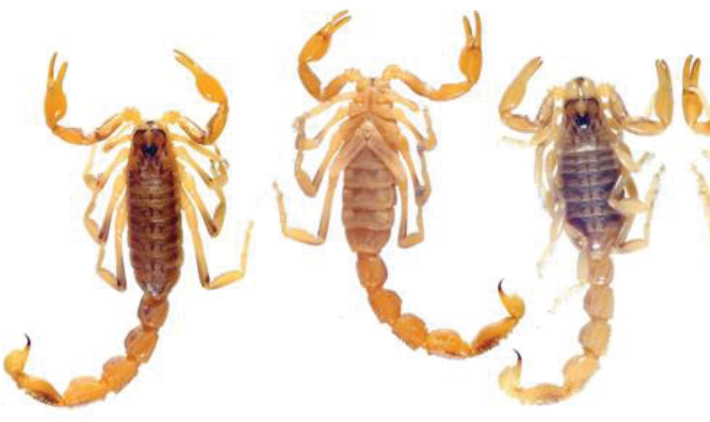

2

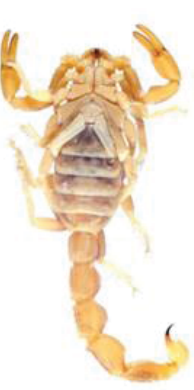

Figure 1,2: Kraepelinia palpator [8], ventral and dorsal views, ô (32 mm), Iran, Kerman Province, Jupar road, $30^{\circ} 07^{\prime} 25^{\prime \prime} \mathrm{N} 57^{\circ} 11^{\prime} 26^{\prime \prime} \mathrm{E}, 1819 \mathrm{~m}$ a.s.I. (Locality No. KE-29),

Figures 3,4: Kraepelinia palpator [8], dorsal and ventral views, $q$ (30 $\mathrm{mm})$, Iran, Kerman Province, Jupar-Kerman road, $30^{\circ} 10^{\prime} 48^{\prime \prime} \mathrm{N} 57^{\circ} 03^{\prime} 02^{\prime \prime} \mathrm{E}, 1788 \mathrm{~m}$ a.s.I. (Locality No. KE-110)

kerman province material examined: Iran, Kerman Province, Kerman Province, Jupar road, 3007'25"N 57¹1'26"E, $1819 \mathrm{~m}$ a.s.l. (Locality No. KE-29), V.2009, 3수 (RRLS), $1{ }^{3}$ (FKCP), leg. Koohpaye, Jamalizadeh and Ebrahimi; Shahdad, Dehghan abad vil., $30^{\circ} 27^{\prime} 20^{\prime \prime} \mathrm{N} 57^{\circ} 49^{\prime} 01^{\prime \prime E}, 334 \mathrm{~m}$ a.s.l. (Locality No. KE-63), V.2009, $13^{`} 2$ (RRLS), leg. Koohpaye, Jamalizadeh and Ebrahimi; Ravar-Mashhad road, $31^{\circ} 21^{\prime} 44^{\prime \prime} \mathrm{N}$ $56^{\circ} 50^{\circ} 08^{\prime \prime E}, 1100 \mathrm{~m}$ a.s.l.(Locality No. KE-84), V.2009, $1{ }^{3}$ (RRLS), leg. Koohpaye, Jamalizadeh and Ebrahimi; Ravar-

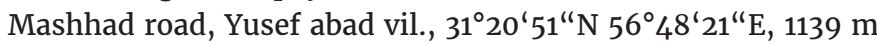
a.s.l. (Locality No. KE-90), V.2009, 1juv. (RRLS), leg. Koohpaye, Jamalizadeh and Ebrahimi; Jupar-Kerman road, $30^{\circ} 11^{\prime} 56^{\prime \prime} \mathrm{N}$ $57^{\circ} 03^{\prime} 18^{\prime \prime E}, 1778 \mathrm{~m}$ a.s.l. (Locality No. KE-109), VI.2009, 1 Љ (RRLS), leg. Koohpaye, Jamalizadeh and Ebrahimi; JuparKerman road, $30^{\circ} 10^{\prime} 48^{\prime \prime} \mathrm{N} 57^{\circ} 03^{\prime} 02^{\prime \prime E}, 1788 \mathrm{~m}$ a.s.l. (Locality No. KE-110), VI.2009, 1 (FKCP), leg. Koohpaye, Jamalizadeh and Ebrahimi; Jupar-Kerman road, $30^{\circ} 07^{\prime} 25^{\prime \prime} \mathrm{N} 57^{\circ} 11^{\prime} 26^{\prime \prime} \mathrm{E}$,

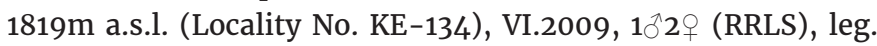
Koohpaye, Jamalizadeh and Ebrahimi.

\section{Polisius persicus (Figures 5-8)}

Distribution:Iran, Sistan \& Baluchistan Province [6], Esfahan Province [9], Ilam Province [10], Kerman Province [7].

kerman province material examined: Iran, Kerman Province, Zehkaloot-Jazmuriyan, $27^{\circ} 4718^{\prime \prime N} \quad 58^{\circ} 35^{\prime} 07^{\prime \prime E}$, $378 \mathrm{~m}$ a.s.l. (Locality No. KE-19), IV.2009, $1{ }^{`} 2$ (RRLS), leg. Koohpaye, Jamalizadeh and Ebrahimi; Zarand, $30^{\circ} 47^{\prime} 42^{\prime \prime} \mathrm{N}$ $56^{\circ} 35^{\prime} 19^{\prime \prime E}, 1678 \mathrm{~m}$ a.s.l. (Locality No. KE-36), V.2009, $1{ }^{3}$ (RRLS), leg. Koohpaye, Jamalizadeh and Ebrahimi; Rafsanjan road, Zarand, $30^{\circ} 32^{\prime} 22^{\prime \prime} \mathrm{N} 56^{\circ} 04^{\prime} 42^{\prime \prime E}, 1547 \mathrm{~m}$ a.s.l. (Locality

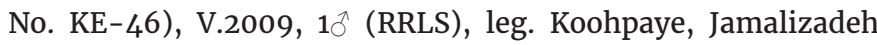
and Ebrahimi; Shahre babak, Marza, 30 $01^{\prime} 43^{\prime \prime} \mathrm{N} 55^{\circ} 05^{\prime} 41^{\prime \prime} \mathrm{E}$, $1789 \mathrm{~m}$ a.s.l. (Locality No. KE-54), V.2009, 1 우 (RRLS), 1 ㅇ im. (FKCP), leg. Koohpaye, Jamalizadeh and Ebrahimi.

Ilam province material examined: Iran, Ilam Prov., Dashte Abbas, Ein Saleh village, $32^{\circ} 25.24^{\prime} \mathrm{N} 47^{\circ} 43.86^{\prime} \mathrm{E}, 182 \mathrm{~m}$ a.s.l. (Locality No. IL-828), X.2007, 1 ㅇ im. FKCP, leg. Navidpour, Masihipour \& Bahrani.

\section{Apistobuthus susanae (Figures 9-12)}

Distribution:Iran, Khoozestan Province [11,12], Ilam Province [10].

kerman province material examined: Iran, Khoozestan

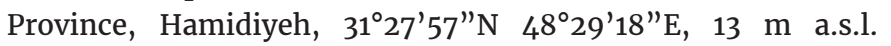
(Locality No. A-Ham-812-1), IX.2007, 18 11우 11juvs RRLS, $1{ }^{\top}$ juv. FKCP, leg. Masihipour \& Navidpour; AhvazMasjedsoleyman road, $31^{\circ} 35^{\prime} 44^{\prime \prime} \mathrm{N}$ 48 $57^{\prime} 19^{\prime \prime E}, 35 \mathrm{~m}$ a.s.l. (Locality No. A-Ma-810), IX.2007, 2318 RRLS, 2 ims. (ふ千ㅇ) FKCP leg. Navidpour \& Masihipour; Ramhormoz road

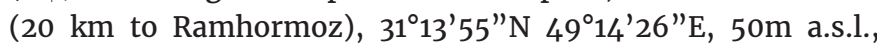
V.2007, 810 RRLS, leg, Masihpour \& Tofigh; Dagh MishanAbdelkhan road, Razihassan village, $31^{\circ} 51^{\prime} 16^{\prime \prime} \mathrm{N} 48^{\circ} 19^{\prime} 07^{\prime} \mathrm{E}$, $42 \mathrm{~m}$ a.s.l., 2007, 10 18 ? RRLS, leg. Habibzadeh, Hayader \& Bahrani; Albaji, Ahvaz-Andimeshk road, $20 \mathrm{~km}$ to Ahvaz,

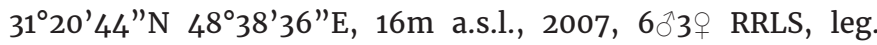
Masihipour, Behani \& Hayader; Ahvaz-Haftgel road (40 km to

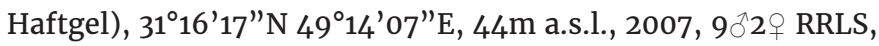
leg. Habibzadeh, Hayader \& Bahrani; Ahvaz-Masjedsoleyman road Zoveyer village, $31^{\circ} 35^{\prime} 20^{\prime \prime} \mathrm{N} 4^{\circ} 57^{\prime} 01^{\prime \prime} \mathrm{E}, 34.5 \mathrm{~m}$ a.s.l., 2007,153

Ilam province material examined: Iran, Ilam Prov., Ein Khosh, $32^{\circ} 24.76^{\prime} \mathrm{N} 47^{\circ} 37.48^{\prime} \mathrm{E}, 130 \mathrm{~m}$ a.s.l. (Locality No. IL-

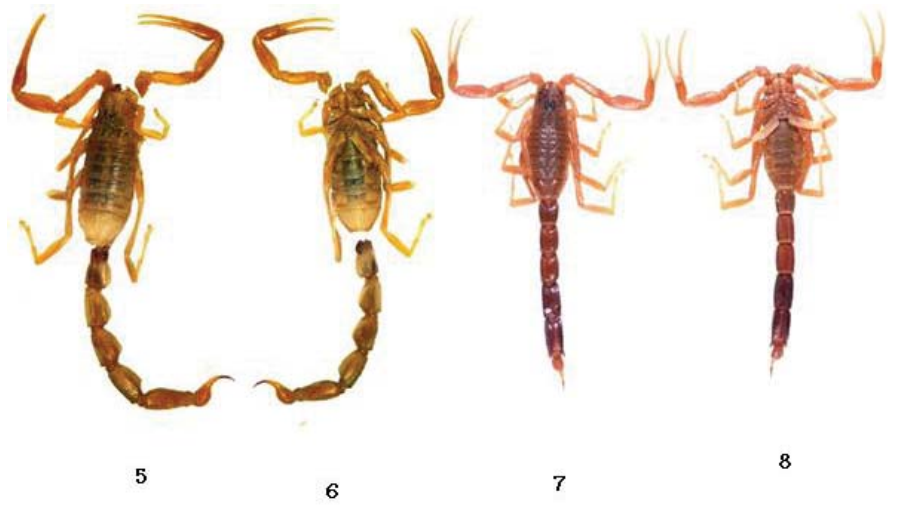

Figure 5,6: Polisius persicus, ventral and dorsal views, $+(41 \mathrm{~mm})$, Iran, Sistan \& Baluchistan Province.

Figures 7,8: Polisius persicus Fet, Capes \& Sissom, 2001 ventral and dorsal views, Jim. (22 mm), Iran, Ilam Province.

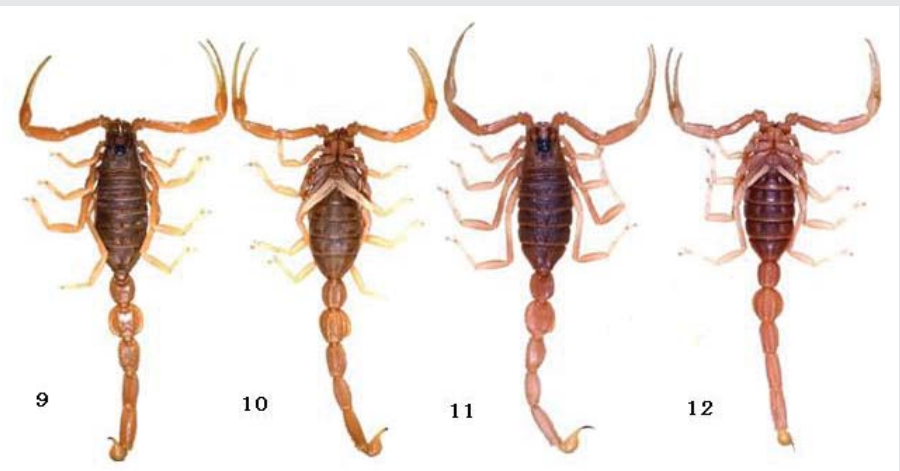

Figure 9,10: Apistobuthus susanae, ventral and dorsal views, $\widehat{\jmath}(76 \mathrm{~mm})$, Iran, Ilam Province.

Figures 11,12: Apistobuthus susanae Lourenço, 1998, dorsal and ventral views, $(69 \mathrm{~mm})$, Iran, Khoozestan Province. 
826), X.2007, $1{ }^{\Uparrow} 1$ 우 FKCP $8{ }^{\jmath} 3$ 울 RRL, leg. Hayader, Masihipour \& Bahrani.

\section{Buthacus macrocentrus (Figures 13-16)}

Distribution:Iran, Bushehr, Hormozgan, Ilam [7,13], and Khoozestan [12] Provinces; Iraq, Israel [14], Jordan [15], Syria [16], Turkey [17].

Khoozestan province material examined: Iran, Khoozestan Province, Ahvaz-Omidiyeh road (40 km to Omidiyeh), $30^{\circ} 37^{\prime} 49^{\prime \prime} \mathrm{N} 49^{\circ} 31^{\prime} 47^{\prime \prime} \mathrm{E}$ (Locality No. 812), V.2007, 1juv. FKCP,

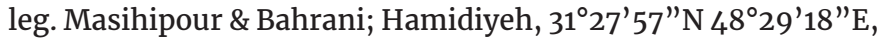

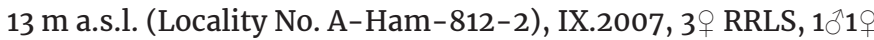
FKCP, leg. Masihipour \& Navidpour; Ahvaz-Masjedsoleyman

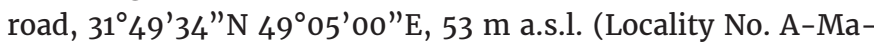
816), X.2007, 4ims. 3juvs. RRLS, leg. Masihipour \& Hayader; Ahvaz-Omidiyeh road (20 km to Omidiyeh), $30^{\circ} 56^{\prime} 12 " \mathrm{~N}$ 49 34'00"E, 53m a.s.l., 2007, $3{ }^{\prime} 5$ 우 1juv. RRLS, leg. Bahrani, Masihipour \& Jahanifard; Chogha Zanbil (zikkurat), $32^{\circ} 00$ ' $55^{\prime \prime} \mathrm{N}$ $4^{\circ} 31^{\prime} 04^{\prime \prime}$ E, $68.5 \mathrm{~m}$ a.s.1., 2007, $4^{\top} 1$ 우 RRLS, leg. Masihipour, Navidpour \& Hayader; Ahvaz-Haftgel road (40km to Haftgel), $31^{\circ} 16^{\prime} 17^{\prime \prime} \mathrm{N} 49^{\circ} 14^{\prime}$ '07"E, $44 \mathrm{~m}$ a.s.l., 2007, 30 RRLS, leg. Habibzadeh, Hayader \& Bahrani; Ahvaz-Omidiyeh road, Chombeh village, $31^{\circ} 11^{\prime} 54^{\prime \prime} \mathrm{N} 49^{\circ} 11^{\prime} 41^{\prime \prime} \mathrm{E}, 44 \mathrm{~m}$ a.s.l., 2007, $33^{\top} 3$ \% RRLS, leg. Masihipour, Navidpour \& Tofigh; Shush (Apadana Palace), $32^{\circ} 10^{\prime} 55^{\prime \prime} \mathrm{N} 48^{\circ} 15^{\prime} 39^{\prime \prime} \mathrm{E}, 75 \mathrm{~m}$ a.s.l., X.2007, $10^{\wedge} 5$ 우 3 juvs. RRLS, leg. Navidpour, Masihipour \& Bahrani.

Ilam province material examined: Iran, Ilam Prov., Dashte Abbas, Ein saleh Village, $32^{\circ} 25.24^{\prime} \mathrm{N} 47^{\circ} 43.86^{\prime} \mathrm{E}, 182 \mathrm{~m}$ a.s.l. (Locality No. IL-828), X.2007, 23우 RRLS 1우 FKCP, leg. Navidpour; Dashte Abbas, Seyed Falhi Village, $32^{\circ} 24.105^{\prime} \mathrm{N}$ $47^{\circ} 36.922^{\prime}$ E, $115 \mathrm{~m}$ a.s.1. (Locality No.IL-829), II.2008, 3 31 우 RRLS, leg. Navidpour, Bahrani \& Hayader.

Bushehr province material examined: Iran, Bushehr Prov., cca $17 \mathrm{~km}$ NW. Bandar-e Genaveh, 10m a.s.l., 29³8'32"N 50²6'56"E, 10 FKCP, 13-14.X.1998, leg. P. Kabátek; Bandar-e Genaveh env., X.2000, $2 \hat{\jmath}$ FKCP, leg. R. Perlík; Delvar,

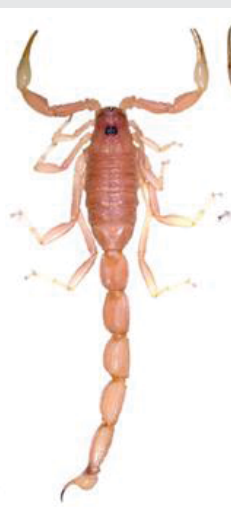

13

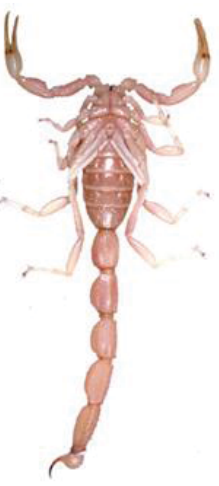

14

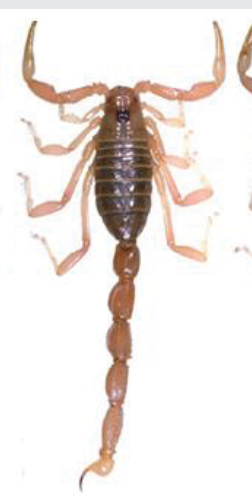

15

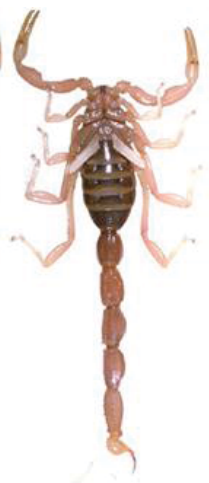

16
Figure 13,14: Buthacus macrocentrus, dorsal and ventral views, $\widehat{\jmath}$ (69 mm), Iran, Khoozestan Province, Hamidiyeh, $31^{\circ} 27^{\prime} 57^{\prime \prime} \mathrm{N} 48^{\circ} 29^{\prime} 18^{\prime \prime} \mathrm{E}, 13 \mathrm{~m}$ a.s.l. (Locality No. A-Ham-812-2), FKCP.

Figures 15,16: Buthacus macrocentrus (Ehrenberg, 1828), dorsal and ventral

views, $q(58 \mathrm{~mm})$, Iran, Khoozestan Province, Hamidiyeh, same locality as in Fig. $56-57$, FKCP. $28^{\circ} 42^{\prime} 59^{\prime \prime} \mathrm{N} 51^{\circ} 04^{\prime} 52^{\prime \prime} \mathrm{E}, 4 \mathrm{~m}$ a.s.l. (Locality No. Bu-20), XI.2007, $182^{2} 2$ 10juvs. RRLS 4juvs. FKCP, leg. Masihipour \& Hayader; Dayer, $27^{\circ} 49^{\prime} 35^{\prime \prime} \mathrm{N} 52^{\circ} 04^{\prime} 44^{\prime \prime} \mathrm{E}, 4 \mathrm{~m}$ a.s.l. (Locality No. Bu-25), XI.2007, 1juv. RRLS, leg. Masihipour, Bahrani \& Habibzadeh; Bushehr to Dayer road, Dero Ahmad village, $27^{\circ} 53^{\prime} 47^{\prime \prime} \mathrm{N} 51^{\circ} 35^{\prime} 51^{\prime \prime} \mathrm{E}, 4 \mathrm{~m}$ a.s.l. (Locality No. Bu-27), XI.2007, $3{ }^{1} 1$ 1juv. RRLS, leg. Masihipour, Hayader \& Habibzadeh; Bushehr to Dayer road, Golestan, 29 ${ }^{\circ} 13^{\prime} 46^{\prime \prime N} 51^{\circ} 19^{\prime} 33^{\prime \prime} \mathrm{E}, 3 \mathrm{~m}$

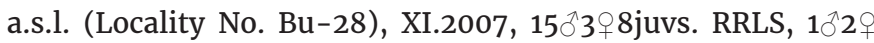
FKCP, leg. Masihipour, Hayder \& Bahrani; Omidiyeh to Genaveh road, 30 $13^{\prime} 42^{\prime \prime} \mathrm{N} 50^{\circ} 12^{\prime} 01^{\prime \prime} \mathrm{E}, 128 \mathrm{~m}$ a.s.l. (Locality No. Bi 805), VI.2007, $1118+22$ juvs. RRLS, leg. Navidpour \& Masihipour; Dailam road, Khite Amareh village, $30^{\circ} 42^{\prime} 52^{\prime \prime} \mathrm{N} 49^{\circ} 44^{\prime} 59^{\prime \prime} \mathrm{E}$, $41 \mathrm{~m}$ a.s.l. (Locality No. OM-801), VII.2007, 132 우 RRLS, leg. Navidpour, Masihipour \& Habibzadeh.

Hormozgan province material examined: Iran, Hormozgan Province, $26^{\circ} 45^{\prime} 57.3^{\prime \prime} \mathrm{N} 54^{\circ} 46^{\prime} 31.4^{\prime \prime E}, 18 \mathrm{~m}$ a.s.l., XI.2008, $40^{1} 4$ 우 (RRLS) , leg. Masihipour \& Hayader; $26^{\circ} 45^{\prime} 14.9^{\prime \prime} \mathrm{N}$ $54^{\circ} 31^{\prime} 44.3^{\prime \prime} \mathrm{E}, 30 \mathrm{~m}$ a.s.l. (Locality No. HO-149), XI.2008, $2{ }^{2}$ ims. (RRLS), leg. Masihipour, Bahrani \& Habibzadeh; $26^{\circ} 46^{\prime} 36.6^{\prime \prime} \mathrm{N} 55^{\circ} 13^{\prime} 59.2$ "E, $5 \mathrm{~m}$ a.s.l. (Locality No. HO-152), XI.2008, $1{ }^{\Uparrow}$ (FKCP) 3ims. (RRLS), leg. Masihipour, Hayader \& Habibzadeh; $45 \mathrm{~km}$. to Khamir Port (Bandare Khamir), $26^{\circ} 50^{\prime} 41.2^{\prime \prime} \mathrm{N} 55^{\circ} 22^{\prime} 06.9$ "E, $17 \mathrm{~m}$ a.s.l. (Locality No. HO-153), XI.2008, 4+4ims. (RRLS) 1ㅇ (FKCP), leg. Masihipour, Hayder \& Bahrani.

\section{Vachoniolus iranus (Figures 17-20)}

Distribution: Iran, Khoozestan Province [12], Ilam Province $[10]$.

Ilam province material examined: Iran, Ilam Prov., Ein Khosh, $32^{\circ} 24.76^{\prime} \mathrm{N} 47^{\circ} 37.48^{\prime} \mathrm{E}, 130 \mathrm{~m}$ a.s.l. (Locality No. IL-826), X.2007, 191im. FKCP, 19im. RRLS, leg. Hayader, Masihipour \& Bahrani.

Khoozestan province material examined: Iran, Khoozestan

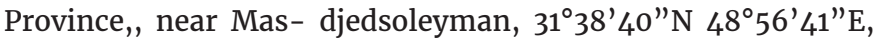
$53 \mathrm{~m}$ a.s.l. (Locality No. A-Ma 806-1), VIII.2007, 8 25+13juvs (holotype and paratypes), leg. Navidpour \& Masihipour; AhvazMasjedsoleyman road, $31^{\circ} 35^{\prime} 44^{\prime \prime} \mathrm{N} 48^{\circ} 57^{\prime} 19^{\prime \prime} \mathrm{E}, 35 \mathrm{~m}$ a.s.l. (Locality No. A-Ma-810), IX.2007, $12{ }^{\top} 27+7$ juvs (paratypes), leg. Navidpour \& Masihipour.

\section{Compsobuthus jakesi (Figures 21-24)}

Distribution:Iran, Bushehr Province [13], Khoozestan Province [12], Ilam Province [10]; Iraq (Kovarik 2003).

Ilam province material examined: Iran, Ilam Prov., Ein Khosh, $32^{\circ} 24.76^{\prime} \mathrm{N} 47^{\circ} 37.48^{\prime} \mathrm{E}, 130 \mathrm{~m}$ a.s.l. (Locality No. IL826), X.2007, $11^{\prime}$ 우 FKCP, leg. Hay- ader, Masihipour \& Bahrani; Dashte Abbas, Ein Saleh Village, $32^{\circ} 25.24^{\prime} \mathrm{N} 47^{\circ} 43.68^{\prime} \mathrm{E}$, $182 \mathrm{~m}$ a.s.l. (Locality No. IL-828), X.2007, $12^{2}$ ㅇ RRLS, leg. Masihipour, Bahrani \& Hayader; Dashte Abbas, Seyed Falhi Village, $32^{\circ} 24.105^{\prime} \mathrm{N} 47^{\circ} 36.922$ 'E, $115 \mathrm{~m}$ a.s.l. (Locality No. IL829), X.2007, $2 q$ RRLS, leg.Masihipour, Habibzadeh \& Hayader; Dehloran, $32^{\circ} 36.36^{\prime} \mathrm{N} 47^{\circ} 20.26^{\prime} \mathrm{E}, 146 \mathrm{~m}$ a.s.l. (Locality No. IL-825), X.2007, 1im. RRLS, leg. Masihipour, Hayader, Habibzadeh \& Bahrani. 


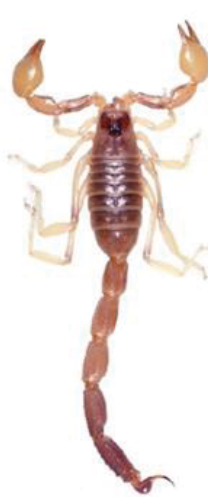

17

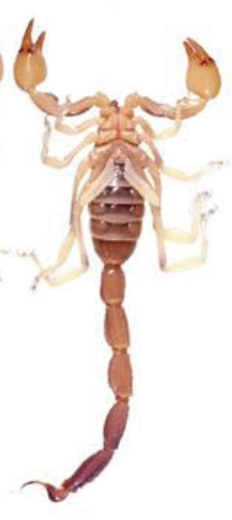

18

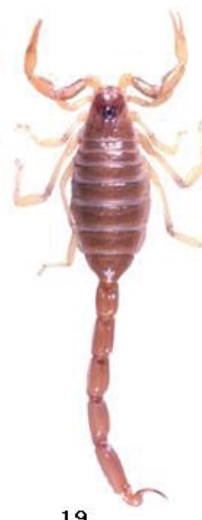

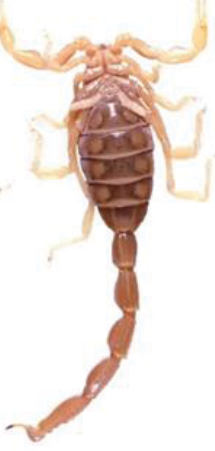

20
Figure 17,18: Vachoniolus iranus ventral and dorsal views, $\delta$ ( $42 \mathrm{~mm})$ holotype, Iran, Khoozestan Province.

Figures 19,20: Vachoniolus iranus, ventral and dorsal views, $\sigma^{\top}(40 \mathrm{~mm})$ allotype, Iran, Khoozestan Province.

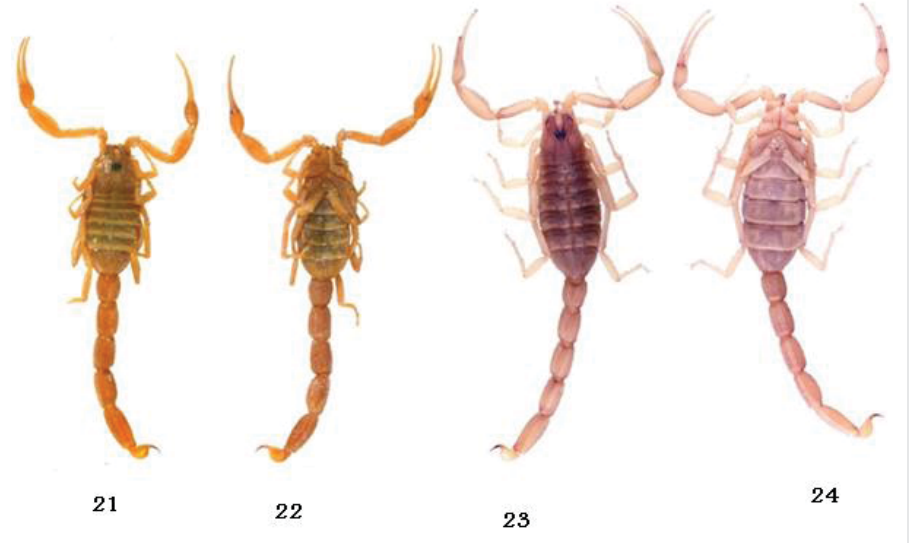

Figure 21,22: Compsobuthus jakesi, dorsal and ventral views, $\widehat{\partial}(28 \mathrm{~mm})$ paratype, Iraq, Najaf Province, Ash-Shabakah (Shabachah, Shabicha), $262 \mathrm{~m}, 31^{\circ} 06^{\prime} \mathrm{N} 43^{\circ} 95^{\prime} \mathrm{E}$. Figures 23,24: Compsobuthus jakesi Kovařik, 2003 [18], dorsal and ventral views, (27 mm), Iran, Khoozestan Province.

Khoozestan province material examined: Iran, Khoozestan Province, Baghmalek, $31^{\circ} 55^{\prime} 17^{\prime \prime} \mathrm{N} 4^{\circ} 22^{\prime} 15^{\prime \prime} \mathrm{E}, 185 \mathrm{~m}$ a.s.l., II.2007, 1 ㅇ RRLS, leg. Masihipour \& Bahrani; Ahvaz-Omidiyeh road , 30³7'49"N 49³1'47"E, (Locality No. 812/803/), V. 2007, 2 \% FKCP, leg. Masihipour \& Bahrani; near Masdjed- soleyman, $31^{\circ} 38^{\prime} 40^{\prime \prime} \mathrm{N} 48^{\circ} 56^{\prime} 41^{\prime \prime} \mathrm{E}, 53 \mathrm{~m}$ a.s.l. (Locality No. A-Ma 806), VIII.2007, 5 $4 \mathrm{ims}$. RRLS, leg. Navidpour \& Masihipour; $45 \mathrm{~km}$ NW of Masdjedsoleyman, Lali, 31 ${ }^{\circ} 18^{\prime} 33^{\prime \prime N} 49^{\circ} 03^{\prime} 39^{\prime \prime E}, 329 \mathrm{~m}$ a.s.l. (Locality No. La-815-3), X.2007, 2ims.(ðㅇ) FKCP, leg. Masihipour \& Hayader; Shush (Apadana Palace), 32 ${ }^{\circ} 10^{\prime} 55^{\prime \prime} \mathrm{N}$ $48^{\circ} 15^{\prime} 39^{\prime \prime}$ E, $75 \mathrm{~m}$ a.s.l., X.2007, 2 \% RRLS, leg. Navidpour, Masihipour \& Bahrani; Ahvaz-Naft Sefid road, $31^{\circ} 27^{\prime} 24^{\prime \prime} \mathrm{N}$ $49^{\circ} 57^{\prime} 37^{\prime \prime E}, 148 \mathrm{~m}$ a.s.l., 2007, 2 ${ }^{\top} 1 \mathrm{juv}$, leg. Masihipour \& Tofigh.

Bushehr province material examined: Iran, Bush- ehr Prov., Bushehr to Dayer road, Dero Ahmad village, $27^{\circ} 53^{\prime} 47^{\prime \prime} \mathrm{N}$ $51^{\circ} 35^{\prime} 51^{\prime \prime} \mathrm{E}, 4 \mathrm{~m}$ a.s.l. (Locality No. Bu-27), XI.2007, 1juv. RRLS, 1 i im. FKCP, leg. Masihipour, Hayader \& Habibzadeh.

\section{Discussion}

Deserts from $25-30 \%$ of the land's surface (polis, 1991), and approximately $20 \%$ of deserts surfaces are caverd by sand (Seely, 1991). Widespread In many deserts (e.g. Namib, Sahara, Karakum), sandy substrates from $64 \%$ of the Sahara, $41 \%$ of the Libyan Desert, $52 \%$ of the Arabian Desert.

Iran in term of climate is part of the Afro-Asian belt of deserts that stretch from the Cape Verde islands off West Africa all the way to Mongolia near Beijing, China.

The geography of Iran consists of a plateau surrounded by mountains and divided into drainage basins. Dasht-e Lut is the largest desert of Iran, 485 kilometers long and 327 kilometers wide, and is considered to be one of the driest places on Earth.

The Dasht-e Kavir or Kavir-e Namak also known as is a extencive desert lying in the middle of the Iranian plateau. It is about 820 kilometers long and 320 kilometers wide making it the Earth's 23rd largest desert. This desert stretches from the Alborz mountains in the north-west to the Dasht-e Lut in the south-east .

A combination between physiological, behavioral and morphological adaptations enable scorpions to tolerate environmental stresses in desert habitats. Scorpions live in sand deserts worldwide. Of 140 known scorpion genera [6], 29 are recorded from sand habitats. Of these, 11 genera exclusively inhabit sand. While some species live exclusively on sand, others have a broader nich that includes other substrates. A four-point scale can be adopted in considering scorpions found only in sandy deserts $[4,19-28]$ :

1) Ultrapsammophiles: species found only in sand habitats with little vegetation.

2) Psammophiles: species found in vegetated sandy areas.

3) Fossorial psammophiles: species that spend almost all their time in burrows.

4) Facultative psammophiles: species that inhabit sand and other substrates, generally these do not possess a highly modified morphology.

Iran has various habitats. Studies showed that over 60 different species in three families (Buthidea, Scorpionidae and Hemiscorpiidae) exists in Iran [7,10,12,13,20-23]. Distribution of scorpions in Iran is the main cause of sorpionism and public health problem. The northern of Iran is rainy and covered by dense forest, but the southern and eastern part geogrificaly is semi-desert and desert areas and frequence of scorpions stings are $>50000$ cases/year.

Our observation and literature suggested that deserts scorpions are important and scorpionism in deserts regions of Iran is serious health problem. This study concluded that six buthidae scorpion species distribute in deserts area that three of them (Apistobuthus suasanae, Butacus macrocentrus and Vachoniolus iranus) are medically dangerous.

Science population density in desert of Iran is comparatively high; hence the knowledge of scorpion distribution in these regions is important. 


\section{References}

1. Fet V, Polis GA, Sissom WD (1998) Life in sandy deserts: the scorpion model. Journal of Arid Environments 39: 609-622. Link: https://bit.ly/3yo9kTB

2. Prendini $L$ (2001) Substratum specialization and speciation in southern African scorpions: the effect hypothesis revisited. 113-138. In Scorpions 2001. In Memoriam Gary A. Polis. (V. Fet \& P.A. Selden, eds.). British Arachnological Society, Burnham Beeches, Buckinghamshire, UK.

3. Polis GA (1990) Ecology. In The Biology of Scorpions. (G.A. Polis, ed.). Stanford University Press, Stanford, California 247-293.

4. Newlands G (1972) Notes on psammophilous scorpions and a description of a new species (Arachnida: Scorpionides). Annals of the Transvaal Museum 27: 241-255. Link: https://bit.ly/3bAyLYk

5. Farzanpay R (1988) A catalogue of the scorpions occuring in Iran, up to january 1986. Revue Arachnologique 8: 33-44. Link: https://bit.ly/3eXqNe8

6. Fet V, Capes EM, Sissom WD (2001) A new genus and species of psammophilic scorpion from eastern Iran (Scorpiones: Buthidae). Pp. 183189. In Scorpions 2001. In Memoriam Gary A. Polis. (V. Fet \& P.A. Selden, eds.). British Arachnological Society, Burnham Beeches, Buckinghamshire, UK. Link: https://bit.ly/3yo9pqn

7. Navidpour S, Ezatkhah M, Kovařik F, Soleglad ME, Fet V ( 2011) Scorpions of Iran (Arachnida, Scorpiones). Part VII. Kerman Province. Euscorpius 131: $1-32$.

8. Birula AA (1903) Beiträge zur Kenntniss der Scorpionenfauna Persiens (Zweiter Beiträg). Bulletin de I'Académie Impériale des Sciences de St. Pétersbourg 19: 67-80.

9. Vignoli V, Kovařík F, Crucitti P (2003) Scorpiofauna of Kashan (Esfahan Province, Iran) (Arachnida: Scorpiones). Euscorpius 9: 1-7. Link: https://bit. ly/3ylNgch

10. Navidpour S, Fet VF, Kovařík ME, Soleglad (2008) Scorpions of Iran (Arachnida, Scorpiones). Part III. Ilam Province. Euscorpius, 69: 1-29. Link: http://bit.ly/37u9v2k

11. Lourenqo WR (1998) A new species of Apistobuthus Finnegan 1932 (Chelicerata, Scorpiones, Buthidae) from Iran. Entomologische Mitteilungen aus dem Zoologischen Museum Hamburg 12: 237-244. Link: https://bit.ly/3hB4uws

12. Navidpour S, Kovařík F, Soleglad ME, Fet V (2008) Scorpions of Iran (Arachnida, Scorpiones). Part I. Khoozestan Province. Euscorpius 65: 1-41. Link: http://bit.ly/34apBMD

13. Navidpour S, Soleglad ME, Fet V, Kovařík F (2008) Scorpions of Iran (Arachnida, Scorpiones). Part II. Bushehr Province. Euscorpius 67: 1-33. Link: https://bit.ly/3osulTc

14. Vachon M (1966) Liste des scorpions connus en Égypte, Arabie, Israël, Liban, Syrie, Jordanie, Turquie, Irak, Iran. Toxicon 4: 209-218. Link: https://bit.ly/3yo9Fpl
15. Pérez Minnocci S (1974) Un inventario preliminar de los escorpiones de la región Paleártica y claves para la identificación de los géneros de la región Paleártica Occidental. Madrid: Universidad Complutense de Madrid, Facultad de Ciencias, Departamento de Zoología, Cátedra de Artrópodos 7: 1-45. Link: https://bit.ly/2QAVHQc

16. Simon E (1892) Liste des Arachnides Recueillis en Syrie par M. le Dr Théod Barrois. Revue Biologique du Nord de la France 5: 80-84.

17. Crucitti P, Vignoli V (2002) Gli Scorpioni (Scorpiones) dell'Anatolia sudorientale (Turchia). Bolletino della Museo Scienze Naturali in Torino 19: 433474.

18. Kovařík F (2003) Eight new species of Compsobuthus Vachon, 1949 from Africa and Asia (Scorpiones: Buthidae). Serket 8: 87-112. Link: https://bit.ly/2T1GfNP

19. Koch LE (1978) A comparative study of the structure, function and adaptation to different habitats of burrows in the scorpion genus Urodacus (Scorpionida, Scorpionidae). Records of the West Australian Museum 6: 119-145. Link: https://bit.ly/3whgNSS

20. Fet V, Lowe G, Braunwalder ME (2000) Catalog of the Scorpions of the World (1758-1998). The New York Entomological Society, New York, 689. Link: https://bit.ly/2QwAhUj

21. Navidpour S, Kovařík F, Soleglad ME, Fet V (2008) Scorpions of Iran (Arachnida Scorpiones). Part IV. Kohgilouyeh \& Boyer Ahmad Province. Euscorpius 74: 1-24. Link: https://bit.ly/3eWsnga

22. Navidpour S, Nayebzadeh HH, Soleglad ME, Fet V, Kovařík F, et al. (2010) Scorpions of Iran (Arachnida, Scorpiones). Part VI. Lorestan Province. Euscorpius 99: 1-23. Link: https://bit.ly/3v6GjtW

23. Navidpour S, Fet V, Kovařik F, Soleglad ME (2012) Scorpions of Iran (Arachnida, Scorpiones). Part VIII. Fars Province. Euscorpius, 139: 1-29. Link: https://bit.ly/3osheH2

24. Fet V (1984) New for the USSR genus and species of scorpions from Badhyz: Kraepelinia palpator (Birula 1903) (Scorpiones, Buthidae). Izvestiya Akademi Nauk Turkmenskoi SSR, Seriya Bio- logicheskikh Nauk (Proceedings of the Academy of Sciences of Turkmen SSR, Series of Biological Sciences), 4: 3743 Link: https://bit.ly/3uZvqd6

25. Habibi T (1971) Liste de Scorpions de l'Iran. Bulletin of the Faculty of Science. Teheran University 2: 42-47.

26. Hadley NF ( 1974) Adaptational biology of desert scorpions. Arachnology 2 : 11-23. Link: https://bit.ly/3bCB4uh

27. kovarík F (2005) Taxonomic position of species of the genus Buthacus Birula 1908 described by Ehrenberg and Lourenço, and description of a new species (Scorpiones: Buthidae). Euscorpius 28: 1-13. Link: http://bit.ly/2Di60mV

28. Kovařík F (1997) Results of the Czech Biological Expedition to Iran. Part 2 Arachnida: Scorpiones with descriptions of Iranobuthus krali gen. n. et sp. n. and Hottentotta zagrosensis sp. n. (Buthidae). Acta Societatis Zoologicae Bohemicae 61:39-52.

Copyright: () 2021 Navidpour SH. This is an open-access article distributed under the terms of the Creative Commons Attribution License, which permits unrestricted use, distribution, and reproduction in any medium, provided the original author and source are credited. 